\title{
Uncertainty inequalities as entanglement criteria for negative partial-transpose states
}

\author{
Hyunchul Nha ${ }^{1, *}$ and M. Suhail Zubairy ${ }^{1,2}$ \\ ${ }^{1}$ Department of Physics, Texas A \& $M$ University at Qatar, Doha, Qatar \\ ${ }^{2}$ Department of Physics and Institute of Quantum Studies, \\ Texas A\& M University, College Station, TX 7r843, USA
}

(Dated: September 18, 2018)

\begin{abstract}
In this Letter, we show that the fulfillment of uncertainty relations is a sufficient criterion for a quantum-mechanically permissible state. We specifically construct two pseudo-spin observables for an arbitrary non-positive Hermitian matrix whose uncertainty relation is violated. This method enables us to systematically derive separability conditions for all negative partial-transpose states in experimentally accessible forms. In particular, generalized entanglement criteria are derived from the Schrödinger-Robertson inequalities for bipartite continuous-variable states.

PACS numbers: 03.65. Ta, 03.65.Ud, 03.67.Mn, 42.50.Dv
\end{abstract}

Quantum mechanics sets a bound on the product of uncertainties of two non-commuting observables at the fundamental level. This uncertainty principle must be fulfilled as a necessary condition for a quantum physical state [1]. In the present Letter, we want to take a deeper view of the role of uncertainty relations by asking: Can the satisfaction of uncertainty relations be regarded as a sufficient condition for a legitimate quantum state?

Our question can be possibly rephrased in many different forms the answers to which may each provide us with valuable insight to quantum physics. One of them is: Can there be any non-positive Hermitian operator that satisfies all uncertainty relations? In fact, this fundamental issue was addressed by a number of people for decades, e.g., in [2], particularly in the phase-space framework of quantum mechanics [3]. Notably, it has been claimed that there is a certain non-positive Hermitian operator that fulfills the uncertainty relations. No one, however, ever presented a conclusive argument, as all considered only a restricted class of uncertainty relations involving the canonical variables in the lowest order. In this Letter, we demonstrate that the satisfaction of uncertainty relations for all pairs of noncommuting observables is indeed sufficient to represent a quantum physical state. In particular, given an arbitrary non-positive Hermitian matrix, we explicitly construct two pseudo-spin observables whose uncertainty relation is violated.

Besides its fundamental importance, our explicit construction has an immediate application as entanglement criteria for the whole class of negative partial-transpose (NPT) states in arbitrary dimensions. When a given state $\rho$ is separable, it is written as a convex sum of product states, $\rho=\Sigma_{i} p_{i} \rho_{1}^{(i)} \otimes \rho_{2}^{(i)} \cdots \otimes \rho_{N}^{(i)}$, where the state $\rho_{j}^{(i)}$ refers to the subsystem $j$. Under partial transposition (PT) for a set of subsystems, a separable state still remains positive, therefore it describes a certain physical state [4]. A number of entanglement criteria have been derived based on PT, and remarkably, all the known criteria for continuous variables $(\mathrm{CVs})$ belong to this category [5, [6, 7, 8, 9, 10]. In particular, the uncertainty relations under PT were employed as the necessary con-

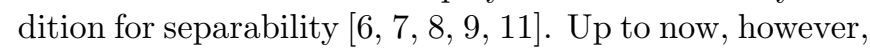
it was not clear to what extent the uncertainty-relationbased approach can detect entangled states. Moreover, given a general mixed entangled state, it is nontrivial to identify the inequality that can be violated by the state.

In this respect, our construction remarkably shows that the uncertainty-relation-based approach is in fact sufficient to detect bipartite entanglement for the whole class of NPT states. More importantly, it enables us to systematically derive entanglement conditions for a given NPT state in experimentally accessible forms. We also show that a weaker form of our nonlinear inequality is equivalent to a well-known entanglement witness formalism [12], providing the witness operator with a physical interpretation as such. We specifically address the generalization of separability conditions based on the SchrödingerRobertson inequalities for CVs 13], which recovers as special cases the previously known criteria, and illustrate their utility in detecting entangled states generated via beam splitter.

Let us start by introducing uncertainty relations. Given two non-commuting observables, $\{A, B\}$, the widely-known Heisenberg uncertainty relation (HUR) reads $\Delta A \Delta B \geq \frac{1}{2}|\langle[A, B]\rangle|$. Less known is the more generalized Schrödinger-Robertson (SR) inequality [13],

$$
\left\langle(\Delta A)^{2}\right\rangle\left\langle(\Delta B)^{2}\right\rangle \geq \frac{1}{4}|\langle[A, B]\rangle|^{2}+\frac{1}{4}\langle\Delta A \Delta B\rangle_{S}^{2},
$$

where the covariance $\langle\Delta A \Delta B\rangle_{S}$ is defined in a symmetric form, $\langle\Delta A \Delta B\rangle_{S} \equiv\langle\Delta A \Delta B+\Delta B \Delta A\rangle$. Clearly, the SR inequality generally provides a stronger bound on the product of uncertainties than the HUR [8]

First, we consider the simplest case of 2-dim systems, which provides us with a valuable insight to the current issue. A general $2 \times 2$ Hermitian matrix $\rho$ is given in a form

$$
\rho=\left(\begin{array}{rr}
a & c \\
c^{*} & b
\end{array}\right)
$$


( $a, b$ : real, $c \equiv c_{r}+i c_{i}$ : complex). For this matrix to represent a physical state, two conditions must be met: (i) $\operatorname{Tr}\{\rho\}=a+b=1$ and (ii) $\operatorname{Det}[\rho]=a b-|c|^{2} \geq 0$. Throughout the present paper, we assume that the trace condition is met, $\operatorname{Tr}\{\rho\}=1$, which can be relaxed later. Then, the only remaining condition is (ii), which turns out to be just a SR-inequality in Eq. (1): Take the angular momentum operators $S_{i}=\frac{\hbar}{2} \sigma_{i}$, where $\sigma_{i}$ is the Pauli spin operator $(i=x, y, z)$. Then, one obtains $\left(\Delta S_{x}\right)^{2}\left(\Delta S_{y}\right)^{2}=\frac{\hbar^{4}}{16}\left(1-4 c_{r}^{2}\right)\left(1-4 c_{i}^{2}\right),\left\langle\left[S_{x}, S_{y}\right]\right\rangle=$ $i \frac{\hbar^{2}}{2}(a-b)$, and $\left\langle\Delta S_{x} \Delta S_{y}\right\rangle_{S}=2 \hbar^{2} c_{r} c_{i}$. On inserting these results to Eq. (11), one immediately finds $a b-|c|^{2} \geq 0$, the condition (ii). Here, the use of the SR inequality is important as one would instead have $a b-|c|^{2}+4 c_{r}^{2} c_{i}^{2} \geq 0$ through the HUR [14, 15].

Therefore, we have the proposition that the physical realizability for 2-dim systems is equivalent to the satisfaction of the single SR inequality between $S_{x}$ and $S_{y}$. At this point, it is worthwhile to observe that the two Hermitian operators $S_{x}$ and $S_{y}$ are represented using the basis states, $|0\rangle$ and $|1\rangle$, as $S_{x}=\frac{\hbar}{2}(|0\rangle\langle 1|+| 1\rangle\langle 0|)$ and $S_{y}=\frac{\hbar}{2 i}(|0\rangle\langle 1|-| 1\rangle\langle 0|)$, which will be used below to construct two pseudo-spin observables to our end.

Now, let us turn our attention to a general Hermitian matrix $\rho$ of arbitrary dimension $N$ with $\operatorname{Tr}\{\rho\}=1$. In general, $\rho$ has the real eigenvalues $\lambda_{i}$ and the corresponding eigenstates $\left|\lambda_{i}\right\rangle(i=1, \cdots, N)$, i.e. $\rho\left|\lambda_{i}\right\rangle=\lambda_{i}\left|\lambda_{i}\right\rangle$, with the orthonormality condition $\left\langle\lambda_{i} \mid \lambda_{j}\right\rangle=\delta_{i j}$. Due to the trace condition, $\operatorname{Tr}\{\rho\}=\Sigma_{i} \lambda_{i}=1$, there always exists at least one positive eigenvalue for $\rho$.

Let us define two pseudo-spin observables $H_{1}$ and $H_{2}$ in the Hilbert space spanned by two eigenstates $\left|\lambda_{1}\right\rangle$ and $\left|\lambda_{2}\right\rangle$ as

$$
\begin{gathered}
H_{1}=\alpha_{1}\left|\lambda_{1}\right\rangle\left\langle\lambda_{2}\left|+\alpha_{1}^{*}\right| \lambda_{2}\right\rangle\left\langle\lambda_{1}\right| \\
H_{2}=\alpha_{2}\left|\lambda_{1}\right\rangle\left\langle\lambda_{2}\left|+\alpha_{2}^{*}\right| \lambda_{2}\right\rangle\left\langle\lambda_{1}\right|,
\end{gathered}
$$

where $\alpha_{1}$ and $\alpha_{2}$ are complex constants. Denoting $x \equiv \operatorname{Re}\left(\alpha_{1} \alpha_{2}^{*}\right)$ and $y \equiv \operatorname{Im}\left(\alpha_{1} \alpha_{2}^{*}\right)$, the commutator $\left[H_{1}, H_{2}\right]=2 i y\left(\left|\lambda_{1}\right\rangle\left\langle\lambda_{1}|-| \lambda_{2}\right\rangle\left\langle\lambda_{2}\right|\right)$ and the anticommutator $\left\{H_{1}, H_{2}\right\}=2 x\left(\left|\lambda_{1}\right\rangle\left\langle\lambda_{1}|+| \lambda_{2}\right\rangle\left\langle\lambda_{2}\right|\right)$ follow together with $H_{i}^{2}=\left|\alpha_{i}\right|^{2}\left(\left|\lambda_{1}\right\rangle\left\langle\lambda_{1}|+| \lambda_{2}\right\rangle\left\langle\lambda_{2}\right|\right)(i=$ 1,2). As $\rho$ is diagonal in the eigenstate basis, $\rho=$ $\operatorname{diag}\left\{\lambda_{1}, \lambda_{2}, \cdots, \lambda_{N}\right\}$, it is straightforward to show

$$
\begin{aligned}
\left\langle\left(\Delta H_{i}\right)^{2}\right\rangle & =\left\langle H_{i}^{2}\right\rangle=\left|\alpha_{i}\right|^{2}\left(\lambda_{1}+\lambda_{2}\right) \\
\left\langle\left[H_{1}, H_{2}\right]\right\rangle & =2 i y\left(\lambda_{1}-\lambda_{2}\right) \\
\left\langle\Delta H_{1} \Delta H_{2}\right\rangle_{S} & =2 x\left(\lambda_{1}+\lambda_{2}\right) .
\end{aligned}
$$

The SR inequality is now reduced to

$$
4 y^{2} \lambda_{1} \lambda_{2} \geq 0 \text {. }
$$

The above inequality is clearly violated if $\lambda_{1} \lambda_{2}<0$, that is, the case that one eigenvalue is positive and the other negative. Therefore, we deduce the fact that the satisfaction of all SR inequalities is sufficient and necessary to endorse a Hermitian matrix of unit trace as a legitimate quantum state.

Note that one could relax the unit-trace condition to any positive trace if the normalization of the matrix were allowed. Furthermore, in Eq. (4), we observe another interesting point for a non-positive Hermitian matrix. If two or more eigenvalues are negative, there exists an observable, $H_{i}(i=1,2)$, whose variance becomes negative. This is of course a clear signature of being unphysical, as all physical observables must have nonnegative variances. Therefore, for a general trace-class Hermitian operator, the satisfaction of all uncertainty relations together with the positivity of variances is sufficient and necessary as a legitimate quantum state.

Aside from its fundamental importance, our explicit construction of the two observables in Eq. (3) can have some practical applications. One of them is the derivation of entanglement condition on demand for an NPT state in arbitrary dimensions. Given a certain $N$-partite state $\rho$, one may wish to determine whether the system possesses bipartite entanglement between two parties, one party $S_{1}$ composed of the subsystems $1, \cdots, j$ and the other $S_{2}$ of the subsystems $j+1, \cdots, N$. Suppose that the state is biseparable as $\rho=\Sigma_{i} p_{i} \rho_{S_{1}}^{i} \otimes \rho_{S_{2}}^{i}$. Then by taking transposition only on $S_{2}$, the density operator transforms as $\rho^{\mathrm{PT}}=\Sigma_{i} p_{i} \rho_{S_{1}}^{i} \otimes\left(\rho_{S_{2}}^{i}\right)^{\mathrm{T}}$, which is still positive definite. In other words, a separable state still remains physical under PT. All the uncertainty relations must therefore be fulfilled as

$$
\begin{aligned}
& \left\langle\left(\Delta H_{1}\right)^{2}\right\rangle_{\mathrm{PT}}\left\langle\left(\Delta H_{2}\right)^{2}\right\rangle_{\mathrm{PT}} \\
& \geq \frac{1}{4}\left|\left\langle\left[H_{1}, H_{2}\right]\right\rangle_{\mathrm{PT}}\right|^{2}+\frac{1}{4}\left\langle\Delta H_{1} \Delta H_{2}\right\rangle_{S, \mathrm{PT}}^{2},
\end{aligned}
$$

where the subscript PT denotes the quantum average over $\rho^{\mathrm{PT}}$ as $\langle\hat{O}\rangle_{\mathrm{PT}} \equiv \operatorname{Tr}\left\{\hat{O} \rho^{\mathrm{PT}}\right\} \quad(\hat{O}:$ arbitrary operator). The inequality (6) must be satisfied by a separable state for any arbitrary operators $H_{1}$ and $H_{2}$, and it thus becomes a separability condition in general [8].

Note that PT preserves the trace and the hermicity of the density operator. Then, for a general entangled state that has some negative eigenvalues under $\mathrm{PT}$, there always exists at least one uncertainty relation that is violated by the NPT state. This means that uncertaintyrelation-based approach to detection of bipartite entanglement is sufficient for the whole class of NPT states. Furthermore, our construction in Eq. (3) enables us to derive an uncertainty inequality as separability condition for a given NPT state as follows.

Given an NPT state $\rho$, one first obtains the eigenvalues and the corresponding eigenstates for the PT density operator. Then take any two eigenstates for $\lambda_{1}>0$ and $\lambda_{2}<0$, and construct the two observables as $H_{1}=$ $\frac{1}{2}\left(\left|\lambda_{1}\right\rangle\left\langle\lambda_{2}|+| \lambda_{2}\right\rangle\left\langle\lambda_{1}\right|\right)$ and $H_{2}=\frac{1}{2 i}\left(\left|\lambda_{1}\right\rangle\left\langle\lambda_{2}|-| \lambda_{2}\right\rangle\left\langle\lambda_{1}\right|\right)$. Then, the SR inequality in Eq. (6) is violated by the given state. Eq. (6) can be further expressed in terms of quantum averages of a normal density operator using the 
general relation $\operatorname{Tr}\left\{\hat{O} \rho^{\mathrm{PT}}\right\}=\operatorname{Tr}\left\{\hat{O}^{\mathrm{PT}} \rho\right\}$, or

$$
\operatorname{Tr}\left\{|i j\rangle\left\langle i^{\prime} j^{\prime}\right| \rho^{\mathrm{PT}}\right\}=\operatorname{Tr}\left\{\left|i j^{\prime}\right\rangle\left\langle i^{\prime} j\right| \rho\right\},
$$

where $|i\rangle$ and $\left|i^{\prime}\right\rangle$ are the basis states for party $S_{1}$, and $|j\rangle$ and $\left|j^{\prime}\right\rangle$ the ones for party $S_{2}$. The inequality (6) can then be expressed in terms of the observables measured with respect to the given state $\rho$, instead of $\rho^{\mathrm{PT}}$.

As an illustration, let us consider a class of tripartite mixed state $\rho_{\mathrm{GHZ}}=p|G H Z\rangle\langle G H Z|+\frac{1-p}{8} I$, where $|G H Z\rangle=\frac{1}{\sqrt{2}}(|000\rangle+|111\rangle)$. With respect to bipartition $\{\mathrm{AB}, \mathrm{C}\}$, the density operator under $\mathrm{PT}$ has two different eigenvalues, $\lambda_{+}=\frac{1+3 p}{8}$ (degenerate) and $\lambda_{-}=\frac{1-5 p}{8}$, thus becomes an NPT state for $p>\frac{1}{5}$. Taking two eigenstates for $\rho_{\mathrm{GHZ}}^{\mathrm{PT}},\left|\lambda_{ \pm}\right\rangle=$ $\frac{1}{\sqrt{2}}(|001\rangle \pm|110\rangle)$, one obtains two observables $H_{1}=$ $\frac{1}{2}\left(\left|\lambda_{+}\right\rangle\left\langle\lambda_{-}|+| \lambda_{-}\right\rangle\left\langle\lambda_{+}\right|\right)=\frac{1}{2}(|001\rangle\langle 001|-| 110\rangle\langle 110|)$ and $H_{2}=\frac{1}{2 i}\left(\left|\lambda_{+}\right\rangle\left\langle\lambda_{-}|-| \lambda_{-}\right\rangle\left\langle\lambda_{+}\right|\right)=$ $\frac{1}{2 i}(|110\rangle\langle 001|-| 001\rangle\langle 110|)$. Then, using the method outlined above, the separability condition is obtained as

$$
\left(4 A_{z}-B_{z}^{2}\right)\left(4 A_{z}-C_{x y}^{2}\right) \geq 16 D_{x y}^{2}+B_{z}^{2} C_{x y}^{2},
$$

where $A_{z} \equiv\left\langle I+\sigma_{1 z} \sigma_{2 z}-\sigma_{1 z} \sigma_{3 z}-\sigma_{2 z} \sigma_{3 z}\right\rangle, B_{z} \equiv\left\langle\sigma_{1 z}+\right.$ $\left.\sigma_{2 z}-\sigma_{3 z}-\sigma_{1 z} \sigma_{2 z} \sigma_{3 z}\right\rangle, C_{x y} \equiv\left\langle\sigma_{1 x} \sigma_{2 x} \sigma_{3 y}+\sigma_{1 x} \sigma_{2 y} \sigma_{3 x}+\right.$ $\left.\sigma_{1 y} \sigma_{2 x} \sigma_{3 x}-\sigma_{1 y} \sigma_{2 y} \sigma_{3 y}\right\rangle$, and $D_{x y} \equiv\left\langle\sigma_{1 x} \sigma_{2 x} \sigma_{3 x}-\right.$ $\left.\sigma_{1 x} \sigma_{2 y} \sigma_{3 y}-\sigma_{1 y} \sigma_{2 x} \sigma_{3 y}-\sigma_{1 y} \sigma_{2 y} \sigma_{3 x}\right\rangle$. The inequalities for the other two bipartitions are obtained simply by taking permutations, and all those separability conditions are violated by the state $\rho_{\mathrm{GHZ}}$ for $p>\frac{1}{5}$, thereby characterizing to some extent its tripartite entanglement.

The above method holds valid for any NPT states in arbitrary dimensions. One can also derive a weaker form of separability condition as

$$
\left\langle H_{1}^{2}\right\rangle_{\mathrm{PT}}\left\langle H_{2}^{2}\right\rangle_{\mathrm{PT}} \geq \frac{1}{4}\left|\left\langle\left[H_{1}, H_{2}\right]\right\rangle_{\mathrm{PT}}\right|^{2} .
$$

The inequality in (9) is weaker than the one in (6), as $\left\langle\left(\Delta H_{i}\right)^{2}\right\rangle_{\mathrm{PT}} \leq\left\langle H_{i}^{2}\right\rangle_{\mathrm{PT}}(i=1,2)$ : If the inequality (9) is violated, so is the inequality (6), but the converse is not always true [16]. It is now straightforward to show that the inequality in (9) is reduced to $\operatorname{Tr}\left\{W_{1} \rho\right\} \operatorname{Tr}\left\{W_{2} \rho\right\} \geq 0$, where $W_{i}=\left|\lambda_{i}\right\rangle\left\langle\left.\lambda_{i}\right|^{\mathrm{PT}}\right.$. Since $\lambda_{1}$ is taken as a positive eigenvalue for $\rho^{\mathrm{PT}}$, it is further reduced to $\operatorname{Tr}\left\{W_{2} \rho\right\} \geq 0$, which is none other than the formalism of the entanglement witness [12]. In other words, the class of entanglement witness operator based on the PT of the entangled state $\left|\lambda_{2}\right\rangle$ can be given a physical interpretation as a weaker form of uncertainty inequality.

Note that it is always more advantageous to use the nonlinear form of the inequality (6) than the linear entanglement witness [12], as the former can be more robust against experimental noises in practice. Moreover, one can also show that any pair of two orthogonal vectors with the condition $\left\langle\lambda_{1}|\rho| \lambda_{1}\right\rangle>0$ and $\left\langle\lambda_{2}|\rho| \lambda_{2}\right\rangle<0$, not necessarily eigenvectors as used so far, can be employed in constructing two pseudo spin observables in Eq. (3). As a consequence, a richer class of separability inequalities may be derived for a given NPT state, which will be addressed elsewhere.

In principle, the above method can also be applied to CVs which can include both finite- and infinitedimensional systems. For finite-dimensional CV states, e.g., the single-photon entangled state $\frac{1}{\sqrt{2}}(|0\rangle|1\rangle+|1\rangle|0\rangle)$ [10], our method can generally work as a practical tool to derive the separability condition. For infinitedimensional CV states, however, except for symmetric states, e.g. EPR state, the computation of the eigenstates may be less tractable than finite-dimensional systems. From another perspective, nevertheless, one can establish an alternative approach still relying on the uncertainty principle. For instance, using a general form of two-mode state $|\lambda\rangle=\Sigma C_{m n}^{\prime} a_{1}^{\dagger m} a_{2}^{\dagger n}|0,0\rangle$ and $|0\rangle\left\langle\left. 0\right|_{i}=: e^{-a_{i}^{\dagger} a_{i}}:(i=1,2\right.$, :: denotes normalordering), two general observables $H_{1}$ and $H_{2}$ can be expressed in terms of the dyadic $\left|\lambda_{1}\right\rangle\left\langle\lambda_{2}\right|=$ $\Sigma \frac{(-1)^{k+k^{\prime}}}{k ! k^{\prime} !} C_{m n} D_{p q} a_{1}^{\dagger(m+k)} a_{1}^{n+k} a_{2}^{\dagger\left(q+k^{\prime}\right)} a_{2}^{p+k^{\prime}}$ and its conjugate $\left|\lambda_{2}\right\rangle\left\langle\lambda_{1}\right|$. Using the relation $\left\langle a_{1}^{\dagger m} a_{1}^{n} a_{2}^{\dagger p} a_{2}^{q}\right\rangle_{\rho^{P T}}=$ $\left\langle a_{1}^{\dagger m} a_{1}^{n} a_{2}^{\dagger q} a_{2}^{p}\right\rangle_{\rho}$ [5, 7], one can derive the separability conditions via the uncertainty relation of $H_{1}$ and $H_{2}$. Remarkably, the satisfaction of them for arbitrary $C_{m n}$ and $D_{p q}$ is sufficient and necessary for the separability of twomode NPT states.

Instead of pursuing a full generalization, left for future work, we demonstrate here that even a little generalization can work out a wide class of important inequalities for CVs. We illustrate the utility of those inequalities by detecting two-mode entanglement generated via a beamsplitter. It is known that a single-mode nonclassical state is sufficient to generate an entangled state via 50:50 beam splitter with the other input in vacuum state [17], however, the entanglement detection at the output is another nontrivial issue to resolve. In fact, it was also conjectured that the whole class of entangled states via beam-splitter is NPT, which is, though, not yet rigorously proved [18]. In this respect, the uncertainty-relation-based approach is very relevant to such class of entangled states.

Let us first define $X_{i}^{(m)} \equiv a_{i}^{\dagger m}+a_{i}^{m}$ and $Y_{i}^{(m)} \equiv$ $-i\left(a_{i}^{\dagger m}-a_{i}^{m}\right)$ for two modes $i=1,2$, and take two Hermitian operators, $H_{1}=X_{1}^{(m)}+X_{2}^{(n)}$ and $H_{2}=Y_{1}^{(m)}+Y_{2}^{(n)}$. The separability condition then follows from the SR inequality, as

$$
\Delta^{2} H_{1} \Delta^{2} \tilde{H}_{2} \geq\left\langle C_{1}^{(m)}+C_{2}^{(n)}\right\rangle^{2}+\left\langle\Delta H_{1} \Delta \tilde{H}_{2}\right\rangle_{S}^{2},
$$

where $\tilde{H}_{2} \equiv Y_{1}^{(m)}-Y_{2}^{(n)}$, and $C_{i}^{(m)} \equiv\left[a_{i}^{m}, a_{i}^{\dagger m}\right](i=1,2)$. For $m=n=1$, a weaker form of the above inequality, i.e., the HUR version ignoring the last term in (10), is reduced to the one derived by Mancini et al. [19] which is known to be stronger than the ones by Duan et al. [20]. 
For another class of inequalities, take $H_{1}=a_{1}^{\dagger m} a_{2}^{\dagger n}+$ $a_{1}^{m} a_{2}^{n}$ and $H_{2}=-i\left(a_{1}^{\dagger m} a_{2}^{\dagger n}-a_{1}^{m} a_{2}^{n}\right)$. Then, one obtains

$$
\begin{aligned}
& \left(\Delta^{2} X_{m n}+\left\langle C_{1}^{(m)} C_{2}^{(n)}\right\rangle\right)\left(\Delta^{2} Y_{m n}+\left\langle C_{1}^{(m)} C_{2}^{(n)}\right\rangle\right) \\
& \geq\left\langle\left[a_{1}^{m} a_{2}^{n}, a_{1}^{\dagger m} a_{2}^{\dagger n}\right]\right\rangle^{2}+\left\langle\Delta X_{m n} \Delta Y_{m n}\right\rangle_{S}^{2}
\end{aligned}
$$

where $X_{m n} \equiv a_{1}^{\dagger m} a_{2}^{n}+a_{1}^{m} a_{2}^{\dagger n}, Y_{m n} \equiv-i\left(a_{1}^{\dagger m} a_{2}^{n}-a_{1}^{m} a_{2}^{\dagger n}\right)$. For $m=n=1$, the HUR version of (11) is reduced to the one in [6, 7], and the SR version to the one in [8]. Furthermore, for general $m$ and $n$, the sum form of HUR version, which is generally weaker than the product form [7], is reduced to the class of inequalities in [10].

The above inequalities in (10) and (11) can successfully detect general two-mode entanglement out of a beam-splitter. Using the inequality (10), one can detect entanglement generated by the whole class of nonclassical states with arbitrary-order amplitude squeezing [21], $\left\langle:\left(\Delta\left(a^{m} e^{-i \phi}+a^{\dagger m} e^{i \phi}\right)\right)^{2}:\right\rangle<0 . \quad m=1$ case refers to the normal quadrature squeezing and $m=2$ to the amplitude-squared squeezing [22]. On the other hand, the inequality (11) detects entanglement by the class of arbitrary-order nonclassical photon statistics, $\left\langle:\left(\Delta a^{\dagger m} a^{m}\right)^{2}:\right\rangle<0(m=1$ : sub-Poissonian $)$. An efficient experimental scheme was proposed to measure general correlation functions of arbitrary orders in 23], which may be suitable for the test of above inequalities.

Finally, let us briefly discuss how the entanglement detection based on the uncertainty relations can be connected to the formalism by Shchukin and Vogel [5]. For a positive Hermitian operator $\rho$, the condition $\operatorname{Tr}\left\{\hat{F}^{\dagger} \hat{F} \rho\right\} \geq 0$ must be met for an arbitrary operator $\hat{F}$. Shchukin and Vogel have derived a hierachy of sufficient and necessary conditions for the positivity under PT by considering a general form of $\hat{F}$. A practical difficulty, though, would be to single out an adequate condition violated by a given state among all of them. On the other hand, we showed that only a special class of the operator $\hat{F}=c_{1} \Delta H_{1}+c_{2} \Delta H_{2}$ is sufficient to detect non-positivity, for which $\operatorname{Tr}\left\{\hat{F}^{\dagger} \hat{F} \rho\right\} \geq 0$ is reduced to the SR inequality (1) by requiring positiveness for any $c_{i}$ 's $(i=1,2)$. Remarkably, our "state-specific" method, which is powerful particularly for finite-dimensional systems, directly relates a single separability condition to any given state in a more physically intuitive term, uncertainty principle.

HN acknowledges Q. Sun, J. Bae, C. Noh, and H. J. Carmichael for helpful discussions. This work is sup- ported by an NPRP grant from the Qatar National Research Fund.*email: hyunchul.nha@qatar.tamu.edu

[1] V. V. Dodonov and V. I. Man'ko, in Invariants and Evolution of Nonstationary Quantum Systems, Proceedings of Lebedev Physics Institute Vol. 183, edited by M. A. Markov (Nova Science, Commack, N.Y., 1989).

[2] O. V. Man'ko et al., Phys. Lett. A 357, 255 (2006); M. de Gosson and F. Luef, ibid. 364, 453 (2007); F. J. Narcowich and R. F. O'Connell, Phys. Rev. A 34, 1 (1986).

[3] J. E. Moyal, Proc. Cambridge Philos. Soc. 45, 99 (1949); H. Nha, Phys. Rev. A 78, 012103 (2008).

[4] A. Peres, Phys. Rev. Lett. 77, 1413 (1996).

[5] E. Shchukin and W. Vogel, Phys. Rev. Lett. 95, 230502 (2005).

[6] G. S. Agarwal and A. Biswas, New J. Phys. 7, 211 (2005).

[7] H. Nha and J. Kim, Phys. Rev. A 74, 012317 (2006).

[8] H. Nha, Phys. Rev. A 76, 014305 (2007).

[9] A. Serafini, Phys. Rev. Lett. 96, 110402 (2006).

[10] M. Hillery and M. S. Zubairy, Phys. Rev. Lett. 96, 050503 (2006).

[11] See also O. Gühne, Phys. Rev. Lett. 92, 117903 (2004) for separability inequalities not relying on PT.

[12] M. Horodecki et al., Phys. Lett. A 223, 1 (1996); O. Gühne and N. Lütkenhaus, Phys. Rev. Lett. 96, 170502 (2006).

[13] E. Schrödinger, Sitzunsber. Preuss. Akad. Wiss., Phys. Math. Kl. 143, 296 (1930); H. Robertson, Phys. Rev. 46, 794 (1934).

[14] However, if one applies an arbitrary unitary operation to the two observables $S_{x}$ and $S_{y}$ and then requires that the HUR must be fulfilled in each case, the same condition (ii) emerges. In this sense, the set of all HURs is unitarily equivalent to a single SR inequality.

[15] See also H. Nha, Phys. Rev. A 76, 053834 (2007).

[16] The condition $\left\langle\left(\Delta H_{i}\right)^{2}\right\rangle_{\mathrm{PT}} \geq 0(i=1,2)$ is assumed in this argument. If, on the other hand, $\left\langle\left(\Delta H_{i}\right)^{2}\right\rangle_{\mathrm{PT}}<0$ holds, it is a signature of quantum entanglement by itself.

[17] J. K. Asboth et al., Phys. Rev. Lett. 94, 173602 (2005); M. S. Kim et al, Phys. Rev. A 65, 032323 (2002); X.-B. Wang ibid. 66, 024303 (2002); M. Hillery and M. S. Zubairy, ibid. 74, 032333 (2006).

[18] J. S. Ivan et al., arXiv:quant-ph/0603255.

[19] S. Mancini et al., Phys. Rev. Lett. 88, 120401 (2002); V. Giovannetti et al., Phys. Rev. A 67, 022320 (2003).

[20] L. M. Duan et al., Phys. Rev. Lett. 84, 2722 (2000).

[21] Z.-M. Zhang et al., Phys. Lett. A 150, 27 (1990).

[22] M. Hillery, Phys. Rev. A 36, 3796 (1987).

[23] E. Shchukin and W. Vogel, Phys. Rev. Lett. 96, 200403 (2006). 